\title{
Resolvent Method Surface Pressures for Airfoil Trailing-Edge Noise Prediction
}

\author{
Georges A. Wagner ${ }^{1}$ and Richard D. Sandberg ${ }^{1}$ \\ ${ }^{1}$ Mechanical Engineering, University of Melbourne, VIC 3010, Australia
}

\begin{abstract}
We present the first resolvent method-based prediction of airfoil trailing-edge noise. Said noise prediction is obtained by using resolvent method pressure predictions as input to Amiet's theory. This framework is tested by comparing the noise prediction to results obtained from using DNS data as input to Amiet's theory. We subsequently analyse whether the resolvent method predicts the incident, scattered or total pressure field, to establish which expression of Amiet's theory to use. The resolvent method is found to capture the total pressure field, even accounting for the interaction of the incident and scattered pressure fields. The analysis is conducted for an acoustically sharp NACA0012 airfoil at zero incidence, and marks an exciting step in the development of a low-cost, physics-driven, hybrid computational aeroacoustics approach.
\end{abstract}

\section{Keywords}

Resolvent; surface pressure; Amiet; trailing-edge noise.

\section{Introduction}

The excessive computational cost of high-fidelity aerodynamic noise predictions precludes their use in engineering design. Low-cost, hybrid methods are therefore predominantly used in industry. In hybrid methods the hydrodynamic and acoustic fields are solved separately. However, low-cost predictions of the hydrodynamic field are usually based on a degree of empiricism. In consequence, accurate, low-cost, hybrid noise predictions are achievable only for configurations similar to the ones they were devised from. We present an airfoil trailing-edge noise prediction framework that has the potential to be physicsdriven and low-cost.

Amiet's classical inviscid theory [2] is an acoustic analogy which translates a given airfoil surface pressure to a far-field noise prediction. There are two different formulations of Amiet's theory of relevance to this work. The first, referred to as Amiet's acoustic analogy or the acoustic analogy, provides the far-field noise from the total pressure difference across the airfoil surface. By total pressure, we mean the sum of the incident and scattered pressure fields. By incident pressure we mean any pressure perturbations convecting downstream towards and over the airfoil trailing-edge (TE). The scattered pressure is defined as any pressure perturbation that results from the flow field scattering at the trailing-edge. This scattered pressure field can radiate upstream, and interfere with the incident pressure field. The scattered field can have a hydrodynamic component and an acoustic component, the actual trailing-edge noise. The second formulation of Amiet's theory, referred to as the modified theory of Amiet, provides the far-field noise solely from the incident pressure field [1]. Both of these were derived in 3D by Amiet, and reformulated to $2 \mathrm{D}$ by Sandberg et al. [8], who numerically verified the expressions three decades after they were formulated. Because these are analytical formulations, combining them with a physics-driven prediction of the hydrodynamic pressure field would provide a physics-driven noise prediction tool.

The focus of this work is therefore on obtaining a low-cost, physics-driven surface pressure prediction to be used in Amiet's theory. This is notoriously difficult due to the cost of highfidelity simulations, the well-reported difficulties in measuring pressure experimentally [5], and the non-trivial nature of the mathematics behind noise generation. We use the resolvent method as formally introduced by McKeon \& Sharma [7]. The latter used a divergence-free formulation, omitting pressure from their analysis of turbulent pipe flow. Luhar et al. [6] retained pressure in their formulation of the resolvent. This lead to impressive rank-1 qualitative predictions of the velocity and pressure fields in turbulent pipe flow. The resolvent method constitutes an exact representation of the Navier-Stokes equations (NSE), and is thus a physics-driven method. The analysis is performed in frequency space, relies on a mean flow as input, and is specific to a given frequency. It has been used to study the flow over airfoils $[11,13,10]$, however mainly for control purposes and accurate pressure predictions were not provided. In fact, Symon et al. [10] remarked that it would be great to see a resolvent pressure prediction for an airfoil. In [12] we presented a first open-flow-configuration prediction of surface pressure from the resolvent method. Here we endeavour to fully validate our noise prediction framework, plugging a resolvent surface pressure prediction into Amiet's theory to obtain an acoustic prediction.

The objective is not to test the validity of Amiet's theory, but to see how a resolvent-based noise prediction compares to a DNSbased noise prediction using Amiet's theory. Further, we wish to establish exactly what the resolvent pressure prediction represents. Is the method capable of providing only one of the incident and scattered pressure fields or is it capable of providing both of these and their interaction, i.e. the total pressure field. Given the two different formulations of Amiet's theory, it is important that we know whether the resolvent surface pressure prediction we obtain corresponds to the incident pressure only, or whether the scattered pressure and its interference with the incident pressure is also accounted for.

\section{Case studied}

We consider 2D flow past a NACA0012 airfoil, at an angle of attack of $0^{\circ}$. The Reynolds number is $R e=u_{\infty} c / v=20,000$, where $u_{\infty}$ is the freestream velocity, $c$ is the airfoil chord length, and $v$ is the kinematic viscosity. The Reynolds number is deliberately kept low so that flow past the airfoil results in a distinct single-frequency wake-shedding mechanism. Consequently we can conclusively compare resolvent and DNS results, without having to combine resolvent analysis results from different frequencies to fully capture the instabilities in the flow. Higher Reynolds number cases, resulting in a more broadband spectrum, mark the next step in the framework development and are currently being studied. Nondimensionalisation is done with respect to the flow-quantities at the freestream and the airfoil chord length.

Direct numerical simulations (DNS) of the configuration are conducted, enabling a direct comparison between low-order approximation (the resolvent method) and the actual flow-field (DNS). The mean-flow that is required as input to the resolvent analysis is also taken from the DNS. While this is not the 
approach that we would like to see used in the ultimate noise prediction framework (requiring a DNS mean flow would defeat the purpose of a low-cost framework), it is essential in this phase of framework development as it guarantees that any difference between the DNS results and the resolvent prediction do not stem from an inaccurate input to the resolvent method. The noise prediction framework is tested at the wake-shedding frequency of the airfoil, where we conduct a resolvent analysis and then use the surface pressure prediction as input to Amiet's acoustic analogy. Subsequently we analyse in more detail whether the resolvent predicts the incident, scattered or total pressure field.

\section{Numerical and Analytical Methods}

\section{Direct Numerical Simulations}

The compressible solver HIPSTAR is used with the overset method of Deuse et al. [3]. The Mach number is set to be very small, $M=0.1$, in order to ensure that compressibility effects have been minimised when comparisons are made to the incompressible resolvent formulation used. A total of $4.1 \times 10^{5}$ grid points are used, with 1200 points along the airfoil surface. At the surface the wall-normal grid point spacing, $\Delta y_{n}$, obeys $\Delta y_{n}^{+} \leq 1$. The NACA0012 airfoil geometry was slightly modified to yield a rounded trailing-edge: at a distance of 0.95 chord length from the leading-edge, the trailing-edge of the original NACA0012 was replaced by a circle arc tangential to the airfoil surface. The resulting geometry is scaled in both directions to enforce unit chord length, with the trailing-edge located at the origin of the coordinate system. This results in a blunt trailingedge thickness $t^{*}$ of approximately $1.4 \%$ chord, i.e. $t^{*}=0.014$. At the trailing-edge, the boundary layer thickness, calculated as per [3], is $\delta^{*}=0.053$.

\section{$\underline{\text { Resolvent Method }}$}

We conduct incompressible resolvent analysis around the temporal mean $\overline{\boldsymbol{u}}(\boldsymbol{x})$. The method stems from introducing a Reynolds decomposition into the incompressible NSE and then Fourier transforming said equations in time, to yield a frequency space equation for the velocity and pressure perturbations. Factorised and written in operator form, said equation reads

$$
\left[\begin{array}{l}
\hat{\boldsymbol{u}}(\boldsymbol{x}, \omega) \\
\hat{p}(\boldsymbol{x}, \omega)
\end{array}\right]=\mathcal{H}(\overline{\boldsymbol{u}}, \omega)\left[\begin{array}{l}
\boldsymbol{I} \\
0
\end{array}\right] \hat{\boldsymbol{f}}(\boldsymbol{x}, \omega),
$$

where $\boldsymbol{u}$ and $p$ are the velocity and pressure, respectively, $\boldsymbol{I}$ is the identity matrix, and $(\hat{*})$ represents a Fourier transformed quantity. Note that Fourier transformation is performed in time only, hence this resolvent formulation is parameterized in terms of the temporal frequency only. The non-linear terms are grouped into $\boldsymbol{f}$, a term dubbed the forcing in the resolvent literature. Therefore

$$
\hat{\boldsymbol{f}}(\boldsymbol{x}, \omega)=\int_{\infty}^{\infty} e^{-i \omega t} \boldsymbol{u}^{\prime}(\boldsymbol{x}, t) \cdot \nabla \boldsymbol{u}^{\prime}(\boldsymbol{x}, t) \mathrm{d} t .
$$

The resolvent operator itself is denoted $\mathcal{H}$, and stems from inverting the global Navier-Stokes operator. To form $\mathcal{H}$ a mean velocity profile is required; resolvent analysis depends on the mean velocity profile provided and is specific to the frequency specified.

Eq. (1) is in input-output form (a concept that stems from control theory), where the resolvent operator represents the transfer function. When written in this form, systems lend themselves to analysis via gain-based optimisation. Here we ask which forcing (input) provides the largest velocity response (output). To answer this we seek to maximise the energetic gain

$$
G_{\max }(\hat{\boldsymbol{f}})=\max _{\hat{\boldsymbol{f}}} \frac{\langle\hat{\boldsymbol{u}}(\boldsymbol{x}, \omega), \hat{\boldsymbol{u}}(\boldsymbol{x}, \omega)\rangle_{u}}{\langle\hat{\boldsymbol{f}}(\boldsymbol{x}, \omega), \hat{\boldsymbol{f}}(\boldsymbol{x}, \omega)\rangle_{f}} .
$$

A Singular Value Decomposition (SVD) is used to establish orthonormal bases for both the system input, the forcing, and the system output, the velocity and pressure response. The SVD provides these modes ranked by order of contribution to the energetic gain. At the frequencies we consider the leading modes have significantly higher energetic content, justifying a rank-1 approximation.

Discretization and numerical implementation of the resolvent approach is implemented using the procedure outlined by Sipp \& Marquet [9], as per Jin [4],. The input and output norms, related to the inner products $\langle\cdot, \cdot\rangle_{u}$ and $\langle\cdot, \cdot\rangle_{f}$, are defined in the former. The grid point distribution used in the DNS is replicated and the domain is discretized using Taylor-Hood finite elements. Velocity is modelled using second order vector elements and pressure is modeled using first order finite elements. In 2D this gives a total of 221,078 vertices in the finite element space. Standard outflow boundary conditions are used at the outlet $(x=7,-7 \leq y \leq 7)$ in the form of $-\hat{p} \boldsymbol{n}+v \nabla \hat{\boldsymbol{u}} \cdot \boldsymbol{n}=0$, incorporated into the variational form ( $\boldsymbol{n}$ being the vector normal to the outlet surface). At the inlet, velocity perturbations are set to zero $(x=-8,-7 \leq y \leq 7)$, and a no-slip and no penetration boundary condition is applied on the airfoil surface.

\section{Amiet's theory}

Amiet's acoustic analogy equation reformulated in 2D as per Sandberg et al. [8] provides the far-field acoustic pressure field from the total pressure difference across the airfoil surface $\Delta p_{\text {tot }}$ :

$$
\mathrm{p}(\boldsymbol{x}, \omega)=\frac{i y \omega b}{4 \beta a} \int_{x_{1}}^{x_{T E}} \Delta p_{t o t}\left(\boldsymbol{x}_{o}, \omega\right) \frac{1}{R} H_{1}^{(2)}\left\{\mu_{0}\left[M\left(x_{o}-x\right)+R\right]\right\} \mathrm{d} x_{o} .
$$

$x_{1}$ is the streamwise coordinate onwards from which the noise sources are considered and hence the lower bound on the integral of the surface pressure difference.

$x_{T E}$ is the streamwise coordinate of the airfoil TE. $\mathrm{p}$ is the 2D acoustic pressure field.

$\boldsymbol{x}_{o}=\left(x_{o}, y_{o}\right)$ is the acoustic observer location.

$\omega$ is the temporal frequency.

$K_{x}=\omega b / U_{c}$ is a non-dimensional wavenumber.

$U_{c}$ is the convection speed of the pressure disturbances.

$b$ is the semi-chord.

$\beta=\sqrt{1-M^{2}}$ is a constant.

$a$ is the speed of sound.

$\mu_{0}=\omega b /\left(c \beta^{2}\right)$ is a reduced frequency.

$R$ is the distance between the observer and the noise source.

$M$ is the Mach number.

$H_{1}^{(2)}$ is a first order Hankel function of the second kind.

There is another version of equation (3), which we refer to as the modified theory of Amiet, which provides the 2-D far-field acoustic pressure field from the incident surface pressure field only [8].

\section{Results}

We have a uniform inflow, zero incidence case with a smooth airfoil surface and no externally introduced perturbation (forcing, trip or other). Therefore, at the shedding frequency, the pressure perturbations causing TE noise stem entirely from the scattered pressure field. There are no pressure perturbations convecting downstream over the airfoil or over the TE, so there is no incident pressure field. The scattered pressure is the total pressure field.

The directivity plots in figure $1 \mathrm{~b}$ are obtained by considering the surface pressure difference over the entire airfoil surface, i.e. integrating over $-1 \leq x \leq 0$ in equation (3). The directivity lobes obtained from the resolvent and DNS surface pres- 
(a)

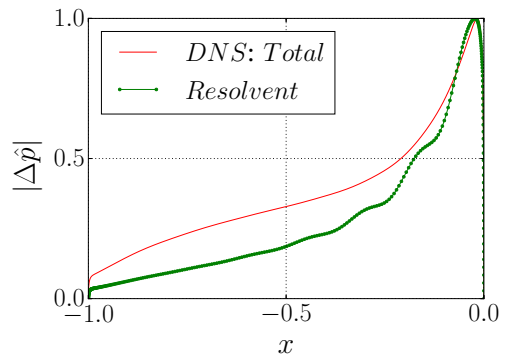

(b)

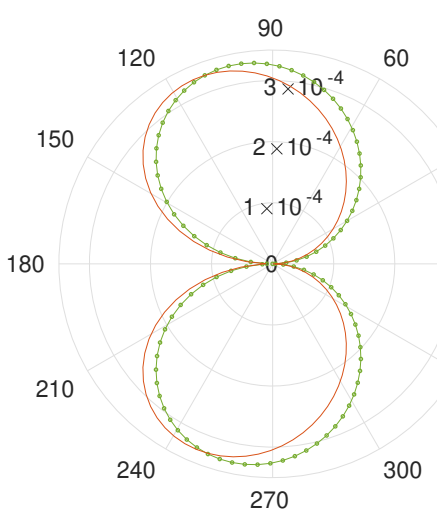

(c)

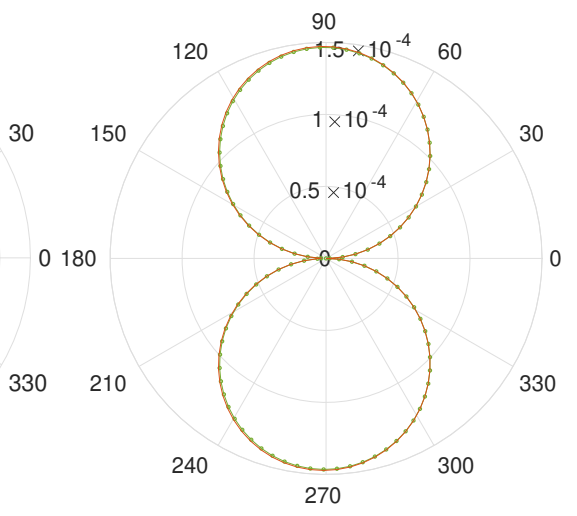

Figure 1. (a) Normalized magnitudes of the DNS and resolvent surface pressure difference across the airfoil surface.

Magnitude of acoustic pressure at $R=2$ from the acoustic analogy, considering (b) the surface pressure over the entire airfoil surface and (c) the surface pressure over $x \in[-0.2,0.0]$.

sures both point upstream. The agreement is promising, yet the resolvent-based noise prediction does not fully capture the direction of the DNS-based noise prediction. This is due to a difference in the surface pressures obtained from the resolvent and the DNS. Figure 1a shows the surface pressure difference from both the resolvent and the DNS, normalized with respect to their respective maximum. Between the leading-edge (at $x=-1)$ and $x \approx-0.2$ the magnitude of the resolvent surface pressure difference is lower than that of its DNS counterpart. In Amiet's theory the trailing-edge (TE) region of the surface pressure is more important than the leading-edge (LE) region, which partly explains the nonetheless impressive agreement in figure $1 \mathrm{~b}$. If we consider only the surface pressures over the region $-0.2 \leq x \leq 0$, then the noise predictions from DNS and resolvent are impressively similar, see figure 1c.

The above results for the wake-shedding frequency indicate that the resolvent is capable of capturing the hydrodynamic scattered pressure field. To investigate whether the resolvent captures solely the scattered pressure field or whether it is equally capable of capturing the incident pressure field, we need an incident pressure perturbation on the airfoil surface.

To this effect we introduce a small-amplitude, single-frequency volume forcing in the DNS. Located on the suction side of the airfoil, downstream of the LE, at $(x, y)=(-0.9,0.05)$, the single frequency volume forcing oscillates at a frequency of $\omega_{F}=8.5 \pi$. Its exact expression can be found in equations (18) and (19) of Sandberg et al. [8]. Because this was explicitly chosen to be a low-amplitude forcing in the unstable region of the flow, it gives rise to laminar Tollmien-Schlichting (TS) waves that convect downstream over the airfoil surface before scattering at the TE, thus constituting an incident pressure field on the airfoil suction side. The resolvent operator, which contains linear dynamics only, should be capable of capturing the effect of this small amplitude forcing. The resolvent energy norm can be interpreted as the question "if we have one unit of energy, how should we force the flow system in order to obtain the most energetic flow perturbation?". At this unstable frequency for the flow system, the answer is a small forcing upstream of the airfoil that gives rise to TS waves, as has been demonstrated on a flat plate [12].

Evidence for the resolvent capturing the incident pressure waves comes from the isocontours of the leading resolvent pressure mode shown in figure 2 . We see the same TS wave pattern as

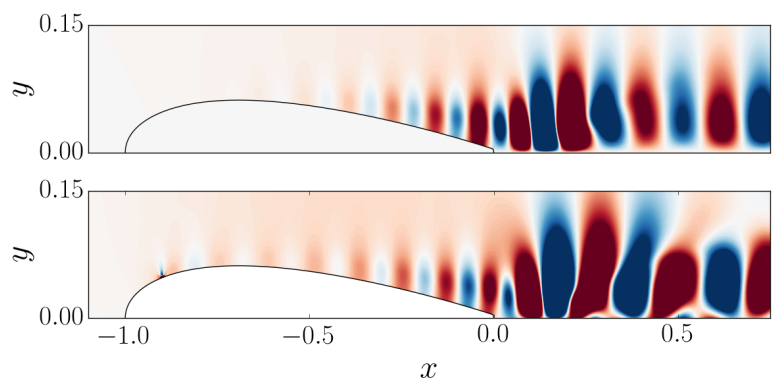

Figure 2. Real part of the pressure field at the frequency $\omega_{F}$ at which a volume forcing has been introduced in the DNS. (Top) Resolvent rank-1 pressure prediction at this unstable frequency; (Bottom) DNS pressure field. The DNS and resolvent values have been normalized by their respective maxima and the contours saturated so that the colorbar range is $[-0.1,0.1]$.

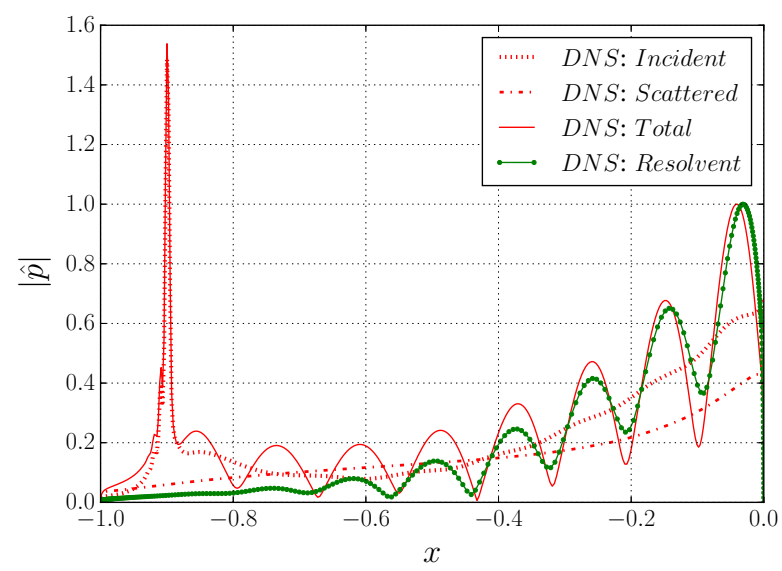

Figure 3. Normalized magnitudes of the suction side DNS incident, scattered and total surface pressure components, as well as the resolvent method surface pressure prediction. The DNS pressure components have all been normalized by the maximum of the total pressure, downstream of the forcing location. The resolvent pressure has been normalized with respect to its own maximum. 
we do in the DNS. The scattered pressure is characteristically out of phase on the suction and pressure sides of the airfoil. By adding the top and bottom surface pressures from the DNS, the incident pressure can thus be isolated. Subtracting the suction side incident pressure from the total pressure on the suction side then yields the scattered pressure. Depicted in figure 3 are the magnitudes of the suction side incident, scattered, and total surface pressures from the DNS, as well as the resolvent method suction side surface pressure. The DNS suction side surface pressure has a spike at the location of the volume forcing. This spike is not present in the resolvent results and was not expected to be, as the resolvent did not have the external forcing applied to it. To draw comparisons between the DNS and resolvent we therefore focus on the region downstream of the forcing location. Unlike the magnitude of the total pressure, the magnitude of the incident pressure does not oscillate, suggesting that the total pressure is a standing wave, whereas the incident pressure is a travelling wave. This means that the downstream convecting TS waves cause a downstream moving pressure field, which interacts with the upstream travelling scattered pressure field, resulting in a stationary pressure field that exhibits a standing wave pattern. Downstream of the half-chord point, the resolvent surface pressure magnitude closely agrees with the qualitative behaviour of the DNS total surface pressure. Having concluded from the analysis at the shedding frequency that the resolvent captures the scattered pressure field, and from looking at the isocontours of pressure on the airfoil that the incident pressure is also captured by the resolvent, the envelope shape of the resolvent surface pressure magnitude now shows that the resolvent seems to capture the total pressure field, i.e. the interaction of the incident and scattered pressure. There is a difference in the pressure amplitude upstream towards the airfoil LE, however it is important to bear in mind that the rank-1 resolvent is an approximation. It follows that Amiet's acoustic analogy expression, equation (3), is the correct formulation of Amiet's theory to use when predicting airfoil trailing-edge noise from resolvent method surface pressure predictions.

\section{Conclusion}

We have presented the first resolvent method-based airfoil TE noise prediction, validating a promising low-cost, physicsdriven noise prediction framework. In studying a case where the incident, scattered and total pressures can be separately assessed and comparing resolvent method pressure predictions to the DNS results, we have established that the resolvent method provides the total pressure field, even accounting for the interaction between the incident and scattered pressure fields. It follows that a resolvent surface pressure prediction can be used as input to Amiet's acoustic analogy theory to obtain far-field trailing-edge noise predictions.

\section{Acknowledgements}

The authors are grateful for the funding provided by the Australian Research Council, Discovery Project Grant \#DP180100898. and the support provided by the Sustaining and strengthening merit-based access to National Computational Infrastructure (NCI) LIEF Grant (LE190100021), facilitated by The University of Melbourne. Further, the computing resources provided at the NCI National Facility systems at the Australian National University through the National Computational Merit Allocation Scheme, supported by the Australian Government, are acknowledged. This work was also partly funded by the U.S. Office of Naval Research Global (ONR Global) under NICOP Grant N62909-17-1-2083. We gratefully acknowledge the support from Dr. Ki-Han Kim (ONG), and Dr. Sung-Eun Kim (ONR Global, Tokyo).

\section{References}

[1] Amiet, R., Effect of the incident surface pressure field on noise due to turbulent flow past a trailing edge, Journal of and Sound Vibration, 57, 1978, 305.

[2] Amiet, R. K., Noise due to turbulent flow past a trailing edge, Journal of Sound and Vibration, 47, 1976, 387-393.

[3] Deuse, M. and Sandberg, R. D., Different noise generation mechanisms of a controlled diffusion aerofoil and their dependence on Mach number, Journal of Sound and Vibration, 115317.

[4] Jin, B., Illingworth, S. J. and Sandberg, R. D., Feedback control of vortex shedding using a resolvent-based modelling approach, Journal of Fluid Mechanics, 897, 2020, A26.

[5] Klewicki, J., Priyadarshana, P. and Metzger, M., Statistical structure of the fluctuating wall pressure and its inplane gradients at high Reynolds number, Journal of Fluid Mechanics, 609, 2008, 195-220.

[6] Luhar, M., Sharma, A. and McKeon, B. J., On the structure and origin of pressure fluctuations in wall turbulence: predictions based on the resolvent analysis, Journal of Fluid Mechanics, 751, 2014, 38-70.

[7] McKeon, B. J. and Sharma, A. S., A critical layer model for turbulent pipe flow, Journal of Fluid Mechanics, 658, 2010, 336-382.

[8] Sandberg, R., Sandham, N. and Joseph, P., Direct numerical simulations of trailing-edge noise generated by boundary-layer instabilities, Journal of Sound and Vibration, 304, 2007, 677-690.

[9] Sipp, D. and Marquet, O., Characterization of noise amplifiers with global singular modes: the case of the leading-edge flat-plate boundary layer, Theoretical and Computational Fluid Dynamics, 27, 2013, 617-635.

[10] Symon, S., Sipp, D. and McKeon, B. J., A tale of two airfoils: resolvent-based modelling of an oscillator versus an amplifier from an experimental mean, Journal of Fluid Mechanics, 881, 2019, 51-83.

[11] Thomareis, N. and Papadakis, G., Resolvent analysis of separated and attached flows around an airfoil at transitional Reynolds number, Physical Review Fluids, 3, 2018, 073901.

[12] Wagner, G. A., Deuse, M., Illingworth, S. J. and Sandberg, R. D., Resolvent analysis-based pressure modeling for trailing-edge noise prediction, in 25th AIAA/CEAS Aeroacoustics Conference, 2019, 2720, 2720.

[13] Yeh, C.-A. and Taira, K., Resolvent-analysis-based design of airfoil separation control, Journal of Fluid Mechanics, 867, 2019, 572-610. 


\section{University Library}

\section{- M M N E R VA A gateway to Melbourne's research publications}

Minerva Access is the Institutional Repository of The University of Melbourne

Author/s:

Wagner, G;Sandberg, R

Title:

Resolvent Method Surface Pressures for Airfoil Trailing-Edge Noise Prediction

Date:

2020-12-11

Citation:

Wagner, G. \& Sandberg, R. (2020). Resolvent Method Surface Pressures for Airfoil TrailingEdge Noise Prediction. Australasian Fluid Mechanics Conference (AFMC), The University of Queensland. https://doi.org/10.14264/6a41fdc.

Persistent Link:

http://hdl.handle.net/11343/294177 